\title{
Die Kinder stehen immer im Zentrum
}

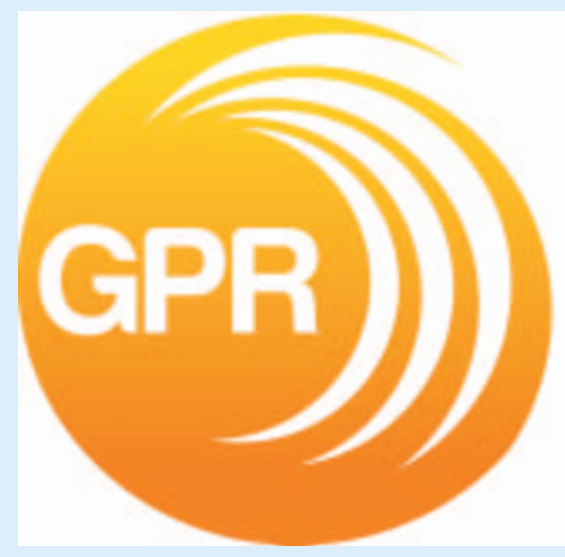

Bereits zum dritten Mal organisiert das Team des Universitätsklinikums Graz die Jahrestagung der GPR. Kongresspräsident Prof. Dr. Erich Sorantin und die Kongresssekretärinnen Dr. Veronika Weiß, Dr. Jana Lacekova und Dr. Eszter Nagy haben ein abwechslungsreiches und umfangreiches Programm auf die Beine gestellt, das neben den klassischen Themen auch einige Spezialfälle beleuchten wird und auch über den Tellerrand der Radiologie hinausschaut.

Herr Professor Sorantin, „Kinderradiologie Reloaded - im Zentrum oder Randerscheinung?“ lautet das Motto der 58. Jahrestagung der GPR. Welche Antwort geben Sie auf diese Frage?

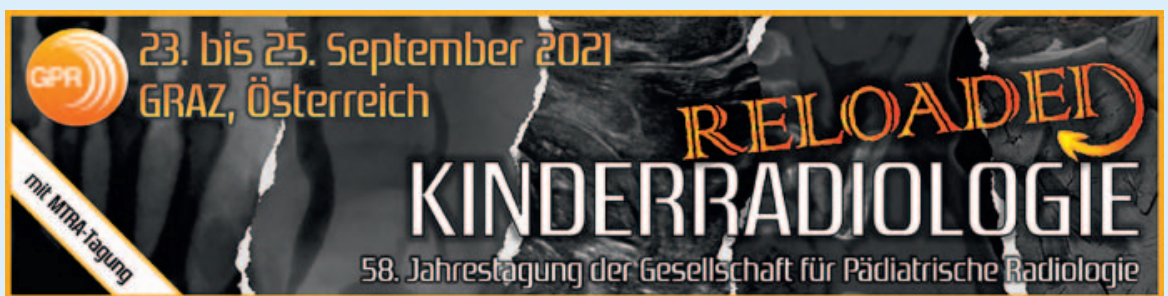

Sorantin Die Kinder stehen immer im Zentrum! Dem gegenüber stehen jedoch nur wenige kinderradiologische Abteilungen, meist konzentriert in den größeren Städten. In Österreich sind wir die einzige kinderradiologische Abteilung an einem akademischen Krankenhaus. Wir bilden das ganze Spektrum ab von der Schwangeren bis zum vollendeten 18. Lebensjahr - Röntgen, Ultraschall, CT, MR. Wir versorgen auch Erwachsene weiter, beispielsweise Patienten mit angeborenen Herzfehlern oder zystischer Fibrose. Alles, was in der Kindheit speziell begonnen hat, versorgen wir weiter. Daher stehen wir im Zentrum der Versorgung des Kindes.

„Zentrum“ bedeutet für uns in Sachen der Jahrestagung, dass wir zentrale Themen der Kinderradiologie in den Fokus nehmen. So begegnen sich klassische Themen wie Asthma, Nierenerkrankungen und bestimmte neurologische Erkrankungen. Daneben gibt es aber auch speziellere Themen, die wir ebenfalls zur Sprache bringen möchten, wie zum Beispiel die Defäkografie. Zentrum und Randerscheinung gehen hier also Hand in Hand. Neben den Krankheitsbildern wollen wir unbedingt in den Fokus rücken, was wir für die Kinder konkret tun können. So wird es einen eigenen Block zum Themenbereich des Frühgeborenen geben, denn diese haben ja ganz eigene Erkrankungen. In einer Session fragen wir uns, was der Neonatologe von uns braucht, und natürlich ist auch der Strahlenschutz immer ein wichtiges Thema. Wir bieten also eine Mischung aus den klassischen Themen, die zudem noch etwas anders gewürzt sind, sowie Themen speziellerer Natur.

Mit "State of the Art and Future Directions“ und „Al \& IT based reporting“ haben Sie komplexe Schwerpunktthemen definiert. Welche übergreifenden Ziele verfolgen Sie mit diesen Themen? 
Sorantin Wir wollen auf der Jahrestagung Wege finden, wie wir die Versorgung der Kinder immer weiter verbessern können. Daher auch das Thema „Future Directions“. Wir wollen versuchen einen Ausblick zu geben, zu schauen, wo die Reise hingeht. Wir stellen vor, was aktuell „hot“ ist in Wissenschaft und Klinik. So haben wir Vorträge über Atemgasanalysen von Intensivpatienten, womit man Infektionen vielleicht früher voraussagen kann. Das andere Schwerpunktthema ist „Artificial Intelligence“. Das wollen wir etwas anders aufziehen, indem wir es zunächst mal gut erklären. Dazu zeigen wir eine Simulation, wie solch ein künstliches System lernt, wann es damit fertig ist und was wir davon erwarten können. Schon gelebte Realität ist die Teleradiologie. Hier werden Akteure aus ihrem Alltag berichten und wir werden das Thema von der Informatikseite und auch juristisch beleuchten.

Welche Themen stehen im Mittelpunkt des kinderradiologischen Refresher- und Intensivkurses?

Sorantin Der Ärztekurs am Samstag thematisiert MR-Sicherheit, Durchleuchtung in der praktischen Anwendung, Kontrastmittel im Kindesalter, was ist beim Kind anders im Röntgen und Tumor vs. Tumor-like lesions. Am Nachmittag wird es Workshops geben zum Lungenultraschall, zu angeborenen Herzfehlern im Thorax-Röntgen oder auch „How to write a scientific paper, abstract or review?“. Ein bunt gemischtes Programm also, um den jungen Kolleginnen und Kollegen ein Rüstzeug mitzugeben einerseits für den täglichen klinischen Alltag, andererseits wissenschaftliche Skills, um für ihre Karriere etwas zu tun.

Das Programm der Jahrestagung richtet sich ja an mehrere Berufsgruppen. Hierzu zählen insbesondere auch die RTs bzw. MTRAs. Welche Angebote halten Sie für diese Gruppe bereit?

Sorantin Hier wird es einen Ländervergleich geben - Deutschland, Österreich, Schweiz. So schauen wir uns die Situation der RTs und MTRAs in den jeweiligen Ländern an, das Arbeitsumfeld und die Kompetenzen. Bei uns in Österreich wird beispielsweise die Frage stark diskutiert, ob RTs selbstständig Ultraschall durchführen

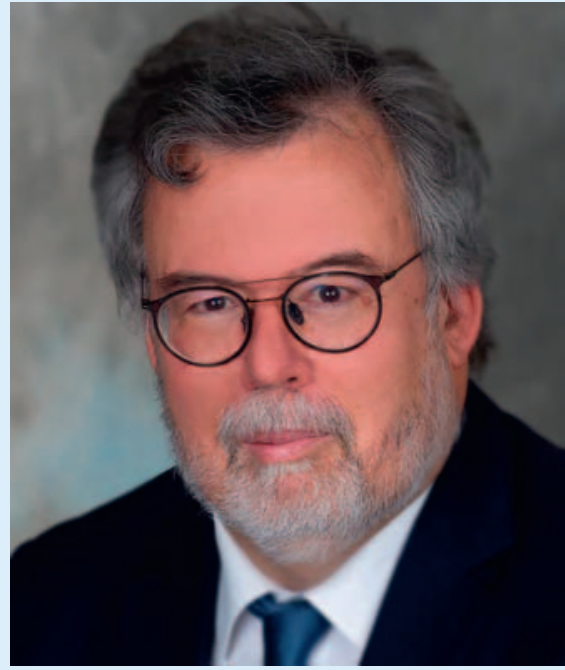

Prof. Dr. Erich Sorantin. Kongresspräsident. (C) privat

dürfen, wenn sie die entsprechenden Lizenzen haben. Oder Herz-CT beim Kind - immer eine Herausforderung. Wie wird das in den jeweiligen Ländern gemacht? Der Schlaganfall bei Kindern ist zwar selten, er kommt aber vor, daher müssen wir daran denken. Auch hier schauen wir in den Ländervergleich: Wer hat das beste Konzept, wo kann man etwas von anderen lernen? Nicht zuletzt geht es um das Kind mit besonderen Bedürfnissen - mit Autismus zum Bespiel oder auch Kinder mit Behinderung. Eine Randerscheinung zwar, aber auch hier ist es wichtig zu wissen, wie man diese kleinen Patienten untersucht.

Macht sich bei Ihnen bereits ein wenig Vorfreude auf die Jahrestagung breit und wenn ja, worauf freuen Sie sich persönlich am meisten?

Sorantin Für mich ist es immer am besten, wenn der Kongress erfolgreich vorbei ist (lacht). Ich freue mich, dass ich für die Kinderradiologie noch einmal etwas auf die Beine stellen kann, was interessiert, was leuchtet. Wir sind eine große Familie in der Kinderradiologie, wir kennen uns alle. Und in dieser Familie wollen wir ein Fest feiern, das uns weiterbringt, das eine bessere Versorgung für die Kinder schafft. Ein Fest, bei dem es auch etwas zu lernen gibt.

Weiß Ich freu mich besonders auf die Vorträge, weil dort sehr viele verschiedene Themen innerhalb kürzester Zeit zusam-

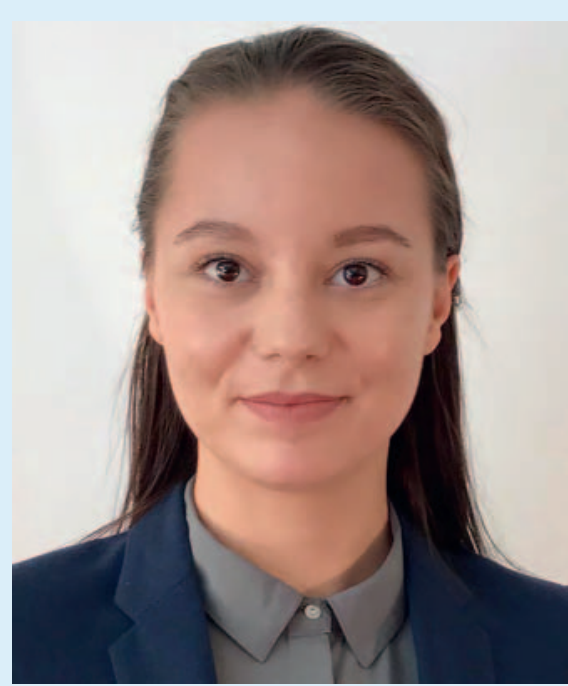

Dr. Veronika Weiss, Kongresssekretärin. (C) privat

menkommen. Aber der Intensivkurs am Samstag ist auf jeden Fall ein Highlight, den werde ich nicht verpassen.

Lacekova Für uns als junge Ärzte ist der Kurs wirklich am interessantesten. Ich war noch nie bei einem Kongress dabei, daher freue ich mich sehr und habe große Erwartungen.

Was werden Sie am ersten Tag nach der Jahrestagung tun?

Sorantin Ich werde einen chinesischen Gast haben, Herrn Liu Jing. Mit ihm, seiner und meiner Frau werde ich auf eine kurze Österreichfahrt gehen.

Weiß Ich werde weiterlernen. Ich bin ja noch in der Ausbildung und bleibe dann im Geschehen drin. Ich baue auf dem auf, was ich auf dem Kongress Neues gelernt habe.

Lacekova Ich werde wahrscheinlich arbeiten, da es ja ein Montag sein wird.

Wie gehen Sie bei der Planung der Jahrestagung mit der aktuellen Pandemie-Situation um? Wird es einen reinen Präsenzkongress geben oder auch digitale Angebote?

Sorantin Wir planen den Kongress als hybride Veranstaltung. Wir werden sehr viele Sessions streamen, sodass man auch online live dabei sein, egal ob die Pandemielage ein Vorortsein möglich macht oder nicht. 


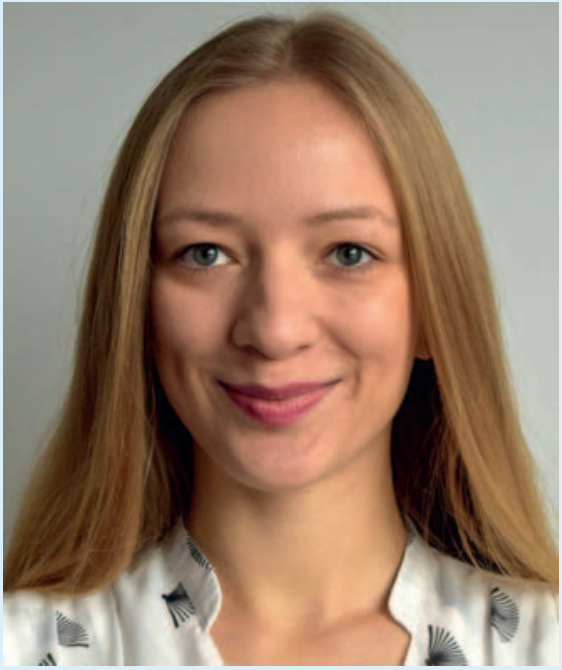

Dr. Jana Lacekova, Kongresssekretärin. (C) privat

Das eröffnet uns vielleicht auch neue Besuchergruppen. Sollten wir - was zu hoffen ist - uns in Graz treffen können, planen wir ein abwechslungsreiches Begleitprogramm. So wird es am sozialen Abend ein Kamingespräch geben mit Caroline Pilhatsch. Sie war Olympiateilnehmerin im Schwimmen im Jahr 2019. Unter dem Titel „An die Spitze schwimmen“ geht es hier um Selbstma-

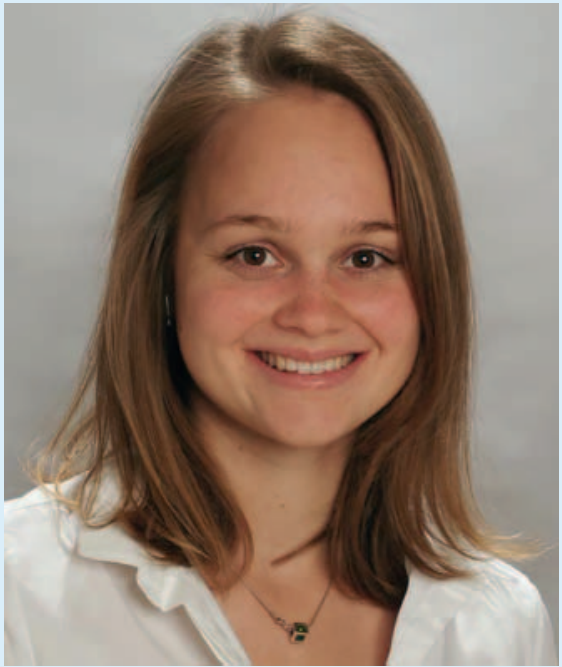

Dr. Ezter Nagy, Kongresssekretärin. (C) privat

nagement, Disziplin und Teamwork. Aus diesem halbstündigen Gespräch wollen wir extrahieren, was daraus für uns als Kinderradiologinnen und Kinderradiologen wichtig ist. Es ist zwar ein ganz anderes Thema, aber auch in der Wissenschaft muss ich mich disziplinieren, muss ich mich so organisieren, dass alles unter einen Hut passt. Und es wird natürlich die Rockband des Kin- derzentrums „Eri \& the Bonebrackers“ an dem Abend spielen. Nicht zuletzt möchte ich erwähnen, dass es auch eine Kinderbetreuung vor Ort geben wird, denn wir möchten dezidiert auch Mütter und Väter dazu ermuntern an der Tagung teilzunehmen. Natürlich alles vorausgesetzt, die Pandemielage lässt es zu. Daumen drücken!

\section{AKTUELLE INFORMATION}

Die GPR-Jahrestagung 2021 wird aktuell als hybrider Kongress - mit Präsenzund Online-Veranstaltungen - geplant. Sollte die Jahrestagung aufgrund der Pandemielage als rein virtueller Kongress stattfinden, finden Sie die Information ab Sommer auf der Homepage www.gpr-jahrestagung.de.

\section{ANMELDUNG GEÖFFNET!}

Ab sofort können Sie sich für die GPRJahrestagung anmelden.

Hier finden Sie die Anmeldung: gpr-jahrestagung.de > ANMELDEN 